\title{
ERRATUM
}

Jorge A. Zegbe • M. Hossein Behboudian

Brent E. Clothier

\section{Responses of 'Petopride' processing tomato to partial rootzone drying at different phenological stages}

Published online: 11 July 2006

(C) Springer-Verlag 2006

\section{Irrig Sci (2006) 24:203-210}

Unfortunately in Table 2 incorrect values for red colours were given. Here is the correct table:

Table 2 Mean fresh weight of fruit $(M F W F)$, dry matter concentration of fruit cortex $(D M C F)$ on a fresh mass basis, fruit water content $(F W C)$ on a fresh mass basis, fruit total soluble solids concentration (TSSC), blossom-end rot (BER), and fruit colour in terms of hue angle $\left(H A^{\circ}\right)$ at green and at firm red stages in response to irrigation treatments

\begin{tabular}{llllllll}
\hline Treatments & $\begin{array}{l}\text { MFWF } \\
(\mathrm{g})\end{array}$ & $\begin{array}{l}\text { DMCF } \\
\left(\mathrm{mg} \mathrm{g}^{-1}\right)\end{array}$ & $\begin{array}{l}\text { FWC } \\
(\%)\end{array}$ & $\begin{array}{l}\text { TSSC } \\
\left({ }^{\circ} \text { Brix }\right)\end{array}$ & $\begin{array}{l}\text { BER } \\
(\%)\end{array}$ & \begin{tabular}{l}
$\mathrm{HA}^{\circ}$ \\
\cline { 3 - 6 }
\end{tabular} \\
\hline FI & $99.0 \mathrm{a}$ & $53.1 \mathrm{~b}$ & $95.0 \mathrm{a}$ & $4.6 \mathrm{~b}$ & $4 \mathrm{~b}$ & $84 \mathrm{a}$ \\
PRD $_{\text {VS-FT }}$ & $97.8 \mathrm{a}$ & $55.0 \mathrm{~b}$ & $94.9 \mathrm{a}$ & $4.8 \mathrm{~b}$ & $8 \mathrm{~b}$ & $84 \mathrm{a}$ & $46 \mathrm{a}$ \\
PRD $_{\text {FT-FS }}$ & $73.8 \mathrm{~b}$ & $64.3 \mathrm{a}$ & $94.2 \mathrm{~b}$ & $5.4 \mathrm{a}$ & $48 \mathrm{a}$ & $76 \mathrm{a}$ \\
PRD $_{\text {FS-H }}$ & $83.3 \mathrm{~b}$ & $61.9 \mathrm{a}$ & $94.2 \mathrm{~b}$ & $5.5 \mathrm{a}$ & $8 \mathrm{~b}$ & $76 \mathrm{a}$ \\
\hline
\end{tabular}

FI daily full irrigation, partial rootzone drying $(P R D)$ during the vegetative stage until the first truss (PRD $\left.\mathrm{VS}_{\mathrm{VT}}\right)$, from the first truss to fruit set $\left(\mathrm{PRD}_{\mathrm{FT}-\mathrm{FS}}\right)$, and from fruit set to harvest $\left(\mathrm{PRD}_{\mathrm{FS}-\mathrm{H}}\right)$. Different letters within columns indicate significant differences by Tukey's Studentised range test at $P \leq 0.05$

The online version of the original article can be found at http://dx.doi.org/10.1007/s00271-005-0018-4.

J. A. Zegbe $\cdot$ M. H. Behboudian Institute of Natural Resources (INR 433), Massey University,

Palmerston North, New Zealand

B. E. Clothier

HortResearch, Private Bag 11030 ,

Palmerston North, New Zealand

Present address: J. A. Zegbe $(\square)$

Campo Experimental Zacatecas, Instituto

Nacional de Investigaciones Forestales,

Agrícolas y Pecuarias, Calera de V.R.,

Apartado Postal No. 18, 98500 Zacatecas, Mexico

E-mail: jzegbe@inifapzac.sagarpa.gob.mx 